\title{
A Fuzzy Rule-Based Feature Construction Approach Applied to Remotely Sensed Imagery
}

\author{
David García $^{1}$ Dimitris Stavrakoudis ${ }^{2}$ Antonio González ${ }^{1}$ Raúl Pérez $^{1}$ John B. Theocharis ${ }^{3}$ \\ ${ }^{1}$ Department of Computational Sciences and AI, ETSIIT, University of Granada 18071, Spain \\ ${ }^{2}$ School of Forestry and Natural Environment, FAFNE, Aristotle University of Thessaloniki 54124, Greece \\ ${ }^{3}$ Department of Electrical and Computer Engineering, Aristotle University of Thessaloniki 54124, Greece
}

\begin{abstract}
The inherent interpretability properties of fuzzy rule-based classification systems (FRBCSs) are undoubtedly one of their major advantages when compared to conventional black-box classifiers. In this paper we present a preliminary study of how the socalled technique of feature construction can prove useful in the context of land cover classification tasks using remotely sensed imagery. The method is integrated into a previously proposed genetic FRBCS (GFRBCS) and applied in a crop classification task using a multispectral satellite image. The experimental analysis shows that feature construction can effectively identify very useful hidden relationships among the initial variables of the problem.
\end{abstract}

Keywords: Genetic fuzzy systems, feature construction, land cover classification, remote sensing, high-dimensional classification tasks.

\section{Introduction}

A typical classification model considers the input variables of the problem as independent sources of information. The employed learning algorithm constructs the model by partitioning the multidimensional feature space (or several subspaces if the classifier supports some sort of embedded feature selection), forming the final decision boundaries. In the context of FRBCSs, the multidimensional relations - as expressed by each fuzzy ruleare defined as overlapping fuzzy hyper-rectangles, although non-axis-parallel classification boundaries can be formed with an appropriate choice of the inference procedure [1].

In certain real-world applications, however, more complex relations exist between the input variables (features). Such relations are typically identified through expert knowledge and after careful examination of the natural properties of the problem at hand. Arguably, it would be desirable to automatically infer more complex relationships among features, through the application of some data mining procedure. The so-called feature construction methods provide an efficient tool for performing such an analysis.
Feature construction [2] can be defined as the process of extracting new features from the original variables of the problem, by means of combination and/or the application of operators or functions. The main objective is to extract hidden useful relationships among them in order to better describe the target concept. The various feature construction methods can be categorized into three main approaches, depending on the way they define the relations and search the feature space [3], namely, those related to:1) decision trees, 2) Inductive Logic Programming (ILP), and 3) genetic algorithms (GA).

In this paper we focus on GA-based feature construction methods. Through the use of GA procedures, it is possible to iteratively extract new features from the set of initial variables. Thus, in each iteration the prediction capability of the model is improved, as new non-trivial and useful information about the problem is being extracted.

Remote sensing classification tasks are typical examples of problems where the input variables are interdependent. The primary information is provided by satellite-borne (or airborne) sensors, which collect the earth's reflected solar radiation in specific portions of the electromagnetic spectrum. It is well-known that different land cover types - and especially plants - absorb specific regions of the spectrum and reflect the remaining radiation [4]. This property has been exploited in the past for devising a number of indices that characterize specific properties of vegetation (greenness, humidity, chlorophyll content, etc.) [5]. For example, the most frequently employed vegetation index is the so-called normalized difference vegetation index (NDVI), which is calculated as the normalized difference between the near-infrared (NIR) and red channels of the image. NDVI relies on the fact that vegetation absorbs the red portion of the spectrum for photosynthetic purposes, but reflects most of the NIR radiation. To this end, green vegetation can be easily differentiated from other land cover types, as it exhibits high NDVI values. This is the primary reason for which all modern sensors targeting at land cover applications bear a NIR channel.

Nevertheless, well-establish vegetation indices typically characterize broad categories of vegetation types and not specific species, despite the advent of hyperspectral spectroscopy, which has led 
to the construction of many new vegetation and chlorophyll content indices [6]. Moreover, equivalent relations for higher-order spectral and textural features - commonly extracted in order to increase classification accuracy when using multispectral images - do not exist. Therefore, the main idea of this work is to apply a GFRBCS that exploits a feature construction methodology on a crop classification task using multispectral satellite imagery. The objective is to identify any new useful relationships between the input variables that can identify the specific crop species of the study area, by analyzing the linguistic interpretation of the fuzzy rules. For this purpose we employ a previously proposed GFRBCS, called NSLV-FR (NSLV Functions and Relations) [7], which provides an effective vehicle for automatic feature construction within a fuzzy rule-based classifier learning framework.

The rest of the paper is organized as follows. Section 2 briefly describes the main components of the base algorithm NSLV. Section 3 explains the major changes performed to the basic NSLV algorithm, in order to incorporate the feature construction technique. Section 4 is devoted to the description of the study area and the dataset formulation, whereas Section 5 presents the experimental results. Finally, Section 6 summarizes the major conclusions derived from the present work.

\section{The learning algorithm NSLV}

NSLV $[8,9]$ is a genetic fuzzy rule learning algorithm that uses a sequential covering strategy [10] together with a GA in order to iteratively extract a single rule. The input of this GA is formed by the consequent variable, the complete set of antecedent variables and the set of examples being the output a single rule covering at least some of the examples. NSLV uses a multi-criteria evaluation function guided by a lexicographical order for selecting the best rule. The different criteria are ordered by their importance level, in order to establish the accuracy and the interpretability level of the rule. The most important one is related to accuracy and it is formulated as the product of the consistency and completeness measures of the rule [11].

NSLV employs a DNF model of fuzzy rules, whereas each rule represents an individual of the population. Thus, a variable takes as value a set of linguistic terms whose members are joined by a disjunctive operator. The GA represents each DNF rule by three different sub-chromosomes or levels (Figure 1):

- The variable level. This level contains a gene for each predictive variable involved in the problem. Each gene represents a real value that it is interpreted as the relevance degree of a predictive variable on the rule. Furthermore, a special gene is added in this level that it is interpreted as activation threshold, that is, the

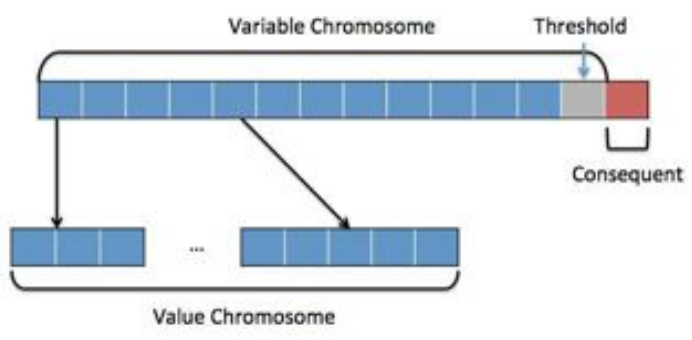

Figure 1: A rule representation in NSLV.

variables for which the associated genes have values less than the threshold are not considered in the antecedent of the rule. Therefore, the use of this level allow us to develop an embedded feature selection process [12].

Two genetic operators are used in this level, two points crossover and real uniform mutation.

- The value level. It is composed of the sequence of assignments to the predictive variables, where each assignment variable/value is represented by a binary string. The complete level is composed by concatenating the binary strings representing the assignments variable/values for all input variables. The assignment of a certain variable will appear in the description of the rule if the value of its associated gene in the variable level permits it.

We use a binary representation and two genetic operators on this level, two points crossover and binary uniform mutation.

- The consequent level. It codes the value of the classification variable of the rule. This level is composed by one gene that is represented by a integer value.

Integer uniform mutation is considered on this level and its value is randomly selected during the initialization of the population.

NSLV uses a modified version of a steady state GA in which the selection process is performed as follows: two individuals of the population are selected and the crossover operators on the respective levels are applied, thus obtaining two new individuals, which are then modified through the respective mutation operators. Subsequently, the two offsprings are evaluated and interchanged with two other individuals of the population. Therefore, in each generation of the genetic process only two new individuals are generated and evaluated. A more detailed description of NSLV can be found in [9].

\section{Including relations and functions in NSLV}

In the introductory section we analyzed the importance of feature construction, specifically in the con- 


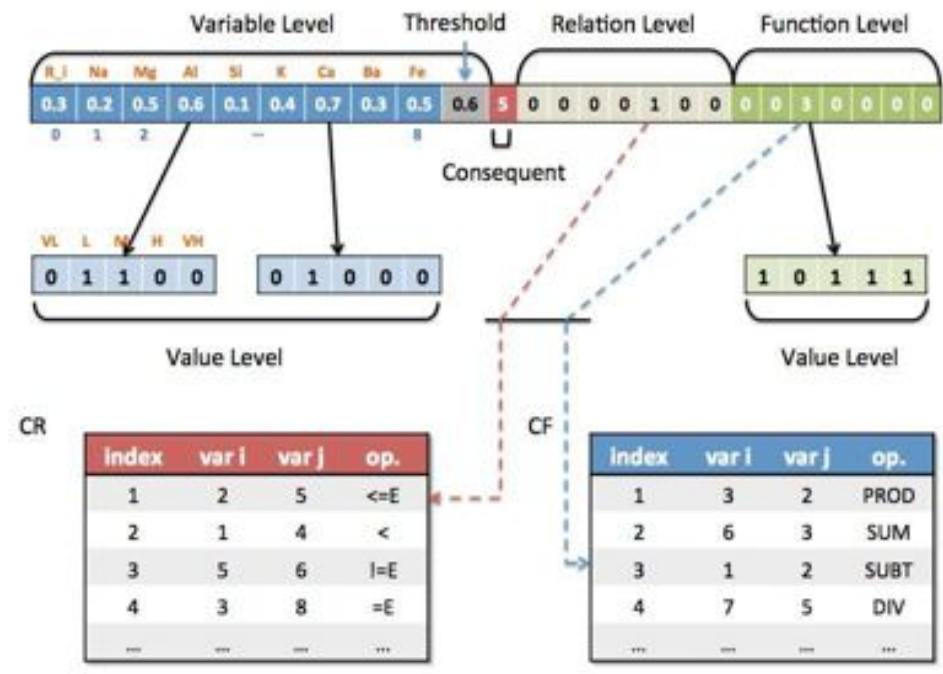

Figure 2: Example of a rule coding.

text of land cover classification using satellite imagery. With this objective in mind, we propose the application of a GFRBCS, namely NSLV-FR, that employs feature construction through relations and functions in the antecedent of fuzzy rules. Relations provide a flexible partitioning of the input space, whereas functions produce new variables with combined information from the original ones.

In order to incorporate this functionality, we introduce four major changes with respect to the base NSLV algorithm:

- A new rule model that supports fuzzy rules in the following form:

IF $X_{2}$ is $A$ and $\left(X_{1}, X_{6}\right)$ are aprox Equal and

$$
\begin{gathered}
P R O D\left(X_{4}, X_{3}\right) \text { is } C \text { THEN } Y \text { is } B \\
\text { with weight } \mathbf{w}
\end{gathered}
$$

where $A$ and $C$ are fuzzy subsets defined on their respective domains, aproxEqual is a fuzzy relation among variables $X_{1}$ and $X_{6}$, $\operatorname{PROD}\left(X_{4}, X_{3}\right)$ is defined as $X_{4} * X_{3}$ and $w$ denotes the rule weight. Every function is considered itself as a new variable, defined in a new fuzzy domain.

- Two structures (acting as an index set) to store the most promising relations and functions to be considered during the learning process: the catalog of relations $(\mathrm{CR})$ and the catalog of functions $(\mathrm{CF})$.

- A new codification of the individuals. Now, each individual incorporates two more levels for handling the information related to relations and functions:

The relation level. This level represents the relation set included in the antecedent part of the rule. Each gene codifies a possible relation of the catalog of relations (up to 10 in the experimental study). It uses an integer coding where 0 indicates "no relation" and any other positive value refers to the index of the relation in the CR.

The function level. This level represents which specific functions from CF will participate in the antecedent part of the rule. The maximum number of functions that can be included in a single rule is defined by a parameter. In the experiments we have considered up to 10 functions for each rule. This function level uses an integer coding where 0 indicates "no function" and any other positive value refers to the index in $\mathrm{CF}$.

- A pruning method through a completeness condition in the fitness function, to establish a threshold representing the minimum percentage of examples (of the class being learned at the specific iteration), that must be covered by a rule.

Figure 2 shows an example of a rule coding. In this paper, we considered three possible relations: less than $(<)$, approximately equal to $(\approx)$, and very different from $(\neq)$. The first one is a binary relation, whereas the other two are fuzzy ones. Moreover, the four typical mathematical operators between two variables (addition, subtraction, multiplication, and division) have been used as possible functions. A thorough description of NSLV-FRalong with the mathematical definition of the aforementioned relations - can be found in [7].

\section{Study area and dataset formulation}

As study area for this work we have selected an agricultural region northwest of Lake Koronia, which is a lake-wetland ecosystem of ecological importance 
in northern Greece $\left(40^{\circ} 41^{\prime \prime} \mathrm{N}, 23^{\circ} 09^{\prime \prime} \mathrm{E}\right)$. An IKONOS bundle image (exhibiting $4 \mathrm{~m}$ spatial resolution in the multispectral channel) was acquired on 7 August 2005 for classification purposes. The IKONOS image has three bands in the visible region of the electromagnetic spectrum, which will be hereafter referred as red $(\mathrm{R})$, green $(\mathrm{G})$ and blue $(\mathrm{B})$ bands/features, and one in the near-infrared (NIR) region.

A portion of $1000 \times 1000$ pixels from the original image was used in this paper. Figure 3(a) depicts a false-color composite of the satellite image, using the NIR, R, and G bands in lieu of the red, green, and blue channels of the color image, respectively. This specific view exploits the vegetation properties discussed in the introduction: green vegetation absorbs visible radiation and reflects NIR; hence it appears red in the color image (only the first channel has high values). Other land cover types also reflect a portion of the visible radiation and so they exhibit different colorings.

The class scheme includes five classes: alfalfa, cereals (primarily wheat), maize, orchards, and artificial surfaces (roads and buildings). After extensive field surveys and subsequent careful photointerpretation, a large number of polygons were identified in the image and assigned to one of the five classes (Figure 3(b)). All labeled pixels (577,316 in total) inside those polygons participate in the testing set. Moreover, 3000 random points (pixels) were selected for the training set. The training patterns were sampled from additional polygons, which do not participate in the testing set.

Due to the limited information provided by the four bands of the initial image, it is customary practice to extract additional higher-order features when dealing with classification tasks involving multispectral images. For this purpose, the following higherorder features were derived from the original bands of the image:

- Spectral transformations. We used the Brightness, Greenness, and Wetness components of the so-called tasseled cap transformation [13], as well as the first three components of the intensity-hue-saturation (IHS) transformation [14] (the saturation component exhibited almost zero variability in the whole image and was therefore not used).

- Textural transformation. As a source of textural information, we applied the gray-level co-occurrence matrix (GLCM) transformation [15]. For each band of the image, four measures from the co-occurrence matrix have been calculated: contrast $(\mathrm{CON})$, angular second moment (ASM), correlation (COR), and homogeneity (HOM). For convenience, we use the notation $<$ measure $>$ : $<$ band $>$ in the following. For example, ASM:R denotes the angular second moment measure calculated from the red band of the original image.

A more thorough description of the study area and the extracted features can be found in [16]. The final feature space of our classification task comprises all 25 aforementioned features (4 initial bands, 5 transformed spectral features, and 16 GLCM measures). Of course, each fuzzy rule in NSLV-FR can also use any derivative relation and function feature from the respective catalog, as described in the previous section.

\section{Experimental analysis}

The objective of this paper is show how the interpretability properties of FRBCSs can be exploited in the remote sensing framework and-most importantly - how the feature construction abilities of NSLV-FR can identify useful new relationships in the specific land cover task considered.

Nevertheless, we first provide a brief comparative from the classification accuracy point of view, in order to validate whether NSLV-FR can achieve comparable performance with other classifiers. In that respect, the comparison with other interpretable classifiers is rather out of scope here. On the contrary, we consider two reference classifiers that have been shown to achieve very high accuracy in many and diverse classification tasks, namely, the support vector machine (SVM) and the random forest (RF) classifiers. The parameters $C$ and $\gamma$ for the SVM classifier (we employed a radial basis function kernel) were determined through a 5 -fold crossvalidation search in the training set, considering a grid of possible values. The final classification model was produced after retraining the SVM with the whole training set and the selected parameters. The same procedure was employed in order to determine the optimal number of trees and random features per tree for the RF classifier. Since NSLV-FR and RF exhibit stochastic characteristics, 20 independent runs were performed using different seeds for the pseudo-random generator (Table 1 shows the results of each single run not only for NSLV-FR, but also for NSLV and two additional algorithms using the feature construction approach, NSLV-R [7], which only handles relations and NSLV-F [7], which works with functions in the antecedent of fuzzy rules).

According to the results shown in Table 1, we can see that the algorithms including the feature construction approach do improve the classification accuracy achieved by NSLV. Moreover, NSLV-FR obtains better results than NSLV-R and NSLV-F, being almost a $10 \%$ and $3 \%$ better than those (talking about average values).

Table 2 reports the comparative results for the three classifiers (NSLV-FR, RF and SVM). For NSLV-FR and RF both average accuracies are reported, along with those exhibited by the best 


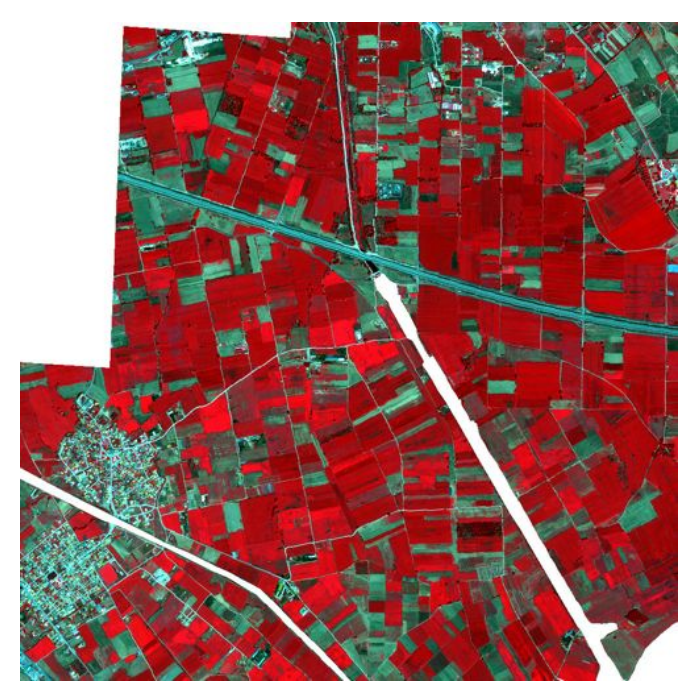

(a)

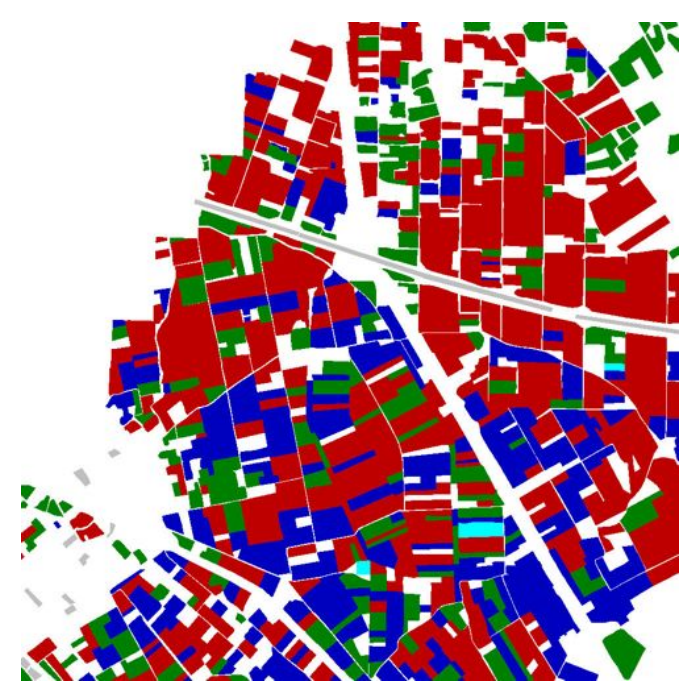

(b)

\section{\begin{tabular}{ll|l|l|l|l|l} 
Alfalfa & Cereals & Maize & Orchards & Artificial \\
\hline
\end{tabular}}

(c)

Figure 3: Study area: (a) pseudo-color composite of the satellite image, (b) the labeled testing polygons, and (c) the respective legend.

\begin{tabular}{||c||c|c|c|c||}
\hline Dataset & NSLV & NSLV-R & NSLV-F & NSLV-FR \\
\hline \hline run1 & $68.9(4)$ & $72.3(3)$ & $75.1(2)$ & $\mathbf{7 8 . 6 ( 1 )}$ \\
run2 & $69.1(4)$ & $70.2(3)$ & $74.8(2)$ & $\mathbf{7 8 . 1 ( 1 )}$ \\
run3 & $41(4)$ & $65.8(3)$ & $77.3(2)$ & $\mathbf{7 7 . 8 ( 1 )}$ \\
run4 & $54.5(4)$ & $65.3(3)$ & $73.7(2)$ & $\mathbf{7 7 . 9 ( 1 )}$ \\
run5 & $43.7(4)$ & $63.4(3)$ & $72.9(2)$ & $\mathbf{7 5 . 3 ( 1 )}$ \\
run6 & $68.9(4)$ & $72.1(3)$ & $\mathbf{7 6 . 8 ( 1 )}$ & $76.2(2)$ \\
run7 & $65.8(4)$ & $68.4(3)$ & $76.1(2)$ & $\mathbf{7 8 . 1 ( 1 )}$ \\
run8 & $70.5(3)$ & $66.2(4)$ & $73.2(2)$ & $\mathbf{7 6 . 9 ( 1 )}$ \\
run9 & $43.5(4)$ & $70.8(3)$ & $\mathbf{7 9 . 3 ( 1 )}$ & $76.6(2)$ \\
run10 & $38.8(4)$ & $62.6(3)$ & $77.3(2)$ & $\mathbf{7 8 . 3 ( 1 )}$ \\
run11 & $69.1(4)$ & $70.5(3)$ & $73.6(2)$ & $\mathbf{7 9 ( 1 )}$ \\
run12 & $47.1(4)$ & $61(3)$ & $65.7(2)$ & $\mathbf{7 6 . 1 ( 1 )}$ \\
run13 & $44.8(4)$ & $68.2(3)$ & $\mathbf{7 6 . 3 ( 1 )}$ & $76.1(2)$ \\
run14 & $43.5(4)$ & $66.4(3)$ & $73.8(2)$ & $\mathbf{7 9 ( 1 )}$ \\
run15 & $44.2(4)$ & $65.5(3)$ & $73.4(2)$ & $\mathbf{7 7 . 1 ( 1 )}$ \\
run16 & $44.7(4)$ & $69(3)$ & $77.6(2)$ & $\mathbf{7 7 . 8 ( 1 )}$ \\
run17 & $38.5(4)$ & $69.9(3)$ & $74.7(2)$ & $\mathbf{7 9 . 2 ( 1 )}$ \\
run18 & $67.1(3)$ & $65.3(4)$ & $76.7(2)$ & $\mathbf{7 8 . 9 ( 1 )}$ \\
run19 & $56.6(4)$ & $72.4(3)$ & $72.8(2)$ & $\mathbf{7 6 . 8 ( 1 )}$ \\
run20 & $54.6(4)$ & $68.5(3)$ & $74.3(2)$ & $\mathbf{7 8 ( 1 )}$ \\
\hline \hline Mean & 53.745 & 67.69 & 74.77 & 77.59 \\
\hline
\end{tabular}

Table 1: Results obtained by the algorithms using feature construction on the previously mentioned dataset. The table shows the accuracy on testing set for each single run (using different seeds).

model, which is defined as the model that achieves the highest accuracy on the testing set. Since SVM's learning algorithm is a deterministic one, only the respective accuracies for the (single) model are reported.

RF exhibits the highest testing accuracy, closely followed by SVM. NSLV-FR displays somewhat lower testing accuracy, although the absolute dif-

\begin{tabular}{l|cc|cc}
\hline \hline \multirow{2}{*}{ Classifier } & \multicolumn{3}{|c}{ Classification accuracy (\%) } \\
\cline { 2 - 5 } & \multicolumn{2}{|c}{ Training } & \multicolumn{2}{c}{ Testing } \\
& Average & Best & Average & Best \\
\hline NSLV-FR & 76.00 & 75.33 & 77.63 & 79.25 \\
RF & 100.0 & 100.0 & 82.67 & 82.89 \\
SVM & \multicolumn{2}{|c}{84.80} & \multicolumn{2}{c}{82.45} \\
\hline \hline
\end{tabular}

Table 2: Training and testing classification accuracies for the compared classifiers. For NSLV-FR and $\mathrm{RF}$ both average and best accuracies are reported.

ference is not that big, especially if we consider the best model. In this analysis we should also take into consideration the much higher complexity of the former two classifiers. Indeed, NSLV-FR creates classification models with 40.6 rules on average, whereas each fuzzy rules comprises an average of 1.67 features. In contrast, the SVM model uses all features and comprises 1436 support vectors, whereas the RF model constructs 100 trees, each of which considers 10 random features. To this end, NSLV-FR's classification accuracy can be relatively considered satisfactory.

In order to exploit NSLV-FR interpretability and feature construction properties, we focus on the best model obtained. As shown in Table 2, this model exhibits a testing accuracy of $79.25 \%$. The primary source of misclassifications stems from the confusion between the alfalfa and maize classes, which are the major green vegetation species in our study area (the confusion matrix is not shown here for reasons of brevity in the presentation). Errors are also observed between the alfalfa and cereals classes, al- 
though to a relatively smaller extent. The latter case is observed because the image was acquired in August, when the cereals crops - most of which were wheat-had been harvested. Hence, sparsely vegetated alfalfa areas are confused with harvested cereal fields, because of the exposed soil. On the other hand, artificial structures can be easily discerned from all other classes, whereas orchards represent a very small percentage of the total land cover.

Therefore, it would be very interesting to analyze the best NSLV-FR model in terms of the features constructed for the discrimination of the alfalfa and maize classes. For this purpose, the fuzzy rules for each class have been sorted in decreasing order with respect to their significance. As a measure of significance for each rule we have employed the (crisp) proportion of positive examples, that is, the number of training patterns that activate the rule and belong to the class described in its consequent, divided by the total number of this class's patterns.

Here we present an example for the maize class. Each feature variable has been uniformly partitioned into fuzzy sets, for which we assign the linguistic labels \{VerySmall, Small, Medium, Large, VeryLarge . The antecedent part of the most significant fuzzy rule comprises two derivative function features:

\section{IF (blue/green) is Large and (brightness + wetness) is VerySmall}

The rule uses a ratio between bands (blue and green) and the sum of two tasseled cap features (brightness and wetness). We should note that neither of these features have some well-established use or expert interpretation in the vegetation indices literature.

Figures 4(c) and 4(d), respectively, depict the grayscale representation of the two new features. For convenience, the study area's pseudo-color composite and the testing polygons have been replicated as the two top subfigures. Areas not belonging to the (testing) reference have been masked out from all images, in order to assist the visual comparison. The fuzzy rule declares that maize exhibits a high blue/green ratio. Indeed, all maize fields in Figure 4(c) have higher grayscale values (brighter areas). At the same time, however, maize is characterized by low values for the brightness+wetness feature (comparatively darker regions in Figure 4(d)). Since NSLV-FR employs the minimum operator for the conjunction of the individual predictive variables, both of these conditions must be satisfied in order for a pattern (pixel in our case) to be characterized as maize.

One way to visualize both features at the same time is to insert them into two channels of a color image and then try to interpret the different colorings produced, based on the description provided by the fuzzy rule. Such false-color composites are very popular in the remote sensing community (in the previous section we have actually provided such an example with the description of Figure 3(a)). In our case, however, an even easier way to produce an aggregate image is to just calculate the rule's activation degrees (memberships) for all image pixels. The result is shown in Figure 4(e). Comparing the latter representation with the reference sites (Figure 4(b)), it becomes apparent that the rule identifies almost all maize fields accurately. Lower (but non-zero) memberships are also observed for a few alfalfa fields as well. This is expected, since the two classes exhibit a strong correlation and are the main source of the misclassifications in the testing set. Nevertheless, most of these regions exhibit membership degrees below 0.5 and as such they can be easily identified.

If we compare the rule activation image and the pseudo-color composite of Figure 4(a) with the reference image, it is evident that the former is far more enlightening with respect to the maize/alfalfa discrimination. Although some alfalfa fields can be singled out from Figure 4(a) as vibrant red, most maize and alfalfa fields are rather indiscriminable. In the previous section we explained that the pseudo-color composite shown in Figure 4(a) relies on the absorption properties of green vegetation in the red and NIR portion of the electromagnetic spectrum. As mentioned in the introduction, NDVI is based on the same principle, being defined as (NIR - Red)/(NIR + Red ), and is ubiquitously employed to identify green vegetation (Figure 4(f)). Again, many alfalfa fields exhibit similar NDVI values with the typical maize ones and cannot be discriminated. To this end, it becomes apparent that NSLV-FR's feature construction ability can prove invaluable for easily discriminating related land cover types in a specific region.

\section{Conclusions}

In this paper we presented the first results from the application of the feature construction concept in the context of land cover classification tasks from remotely sensed imagery. For this purpose, we exploited the advantages of the NSLV-FR classifier, which effectively embeds a feature construction procedure within a powerful genetic fuzzy rule-base learning framework. The experimental analysis was performed on a challenging crop classification task, using a very high resolution satellite image. The linguistic interpretation of the fuzzy rule base resulted in interesting conclusions, since we have been able to visually discriminate spectrally similar vegetation species. As a future work, we will try to discover similar relationships for other land cover types and - most importantly - we will try to apply the methodology in hyperspectral imagery. 


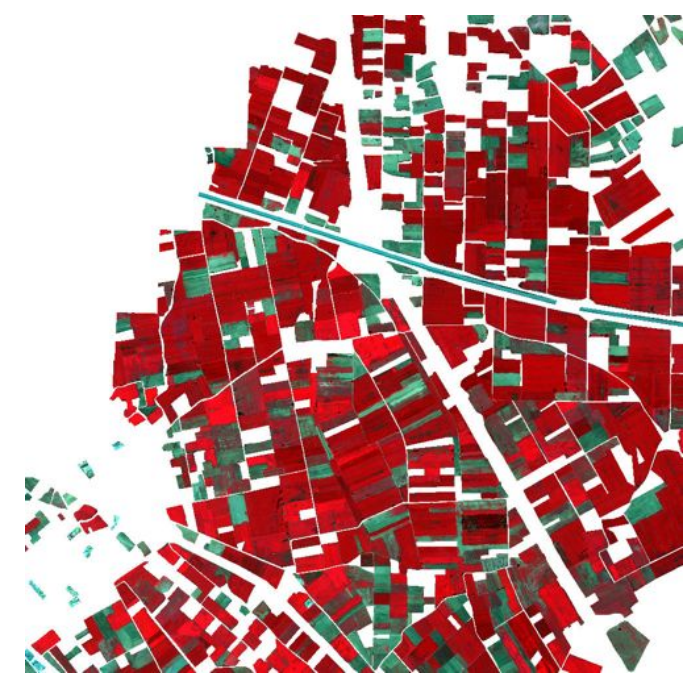

(a) Satellite image pseudo-color composite

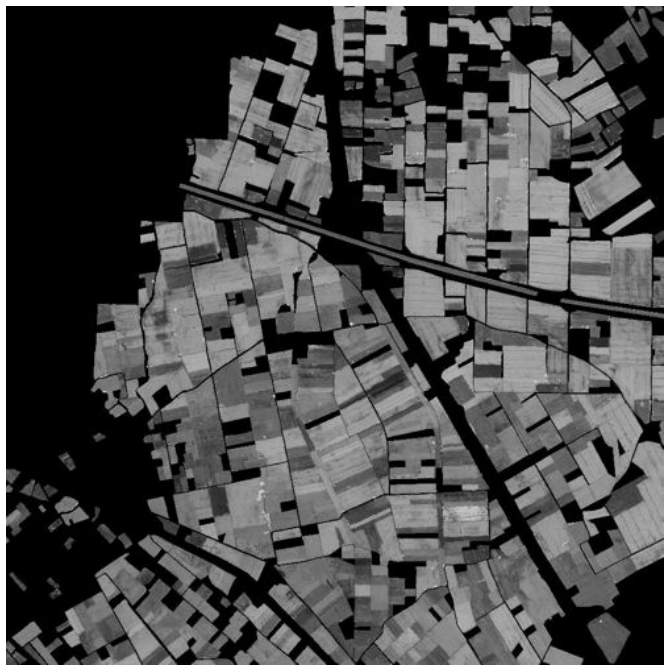

(c) (Blue/Green) feature
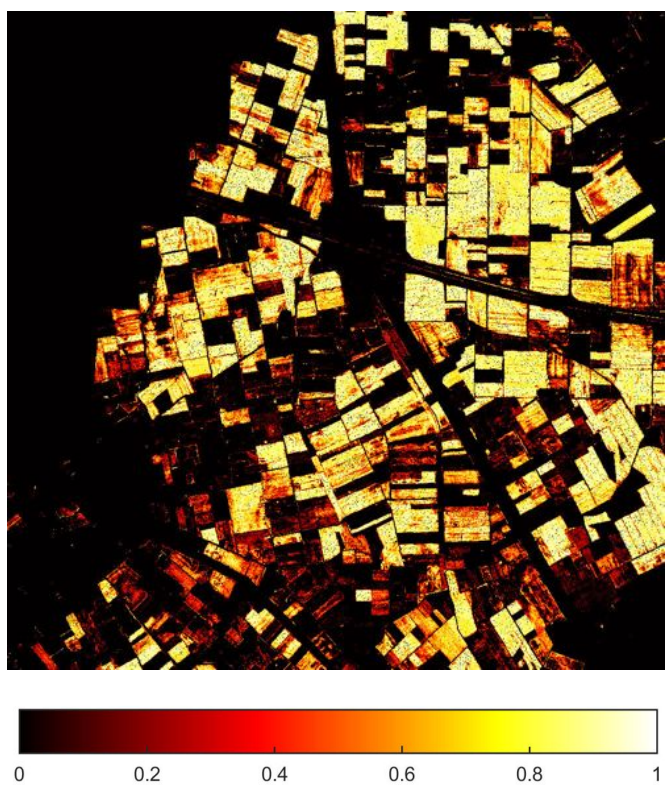

(e) Rule activation degrees

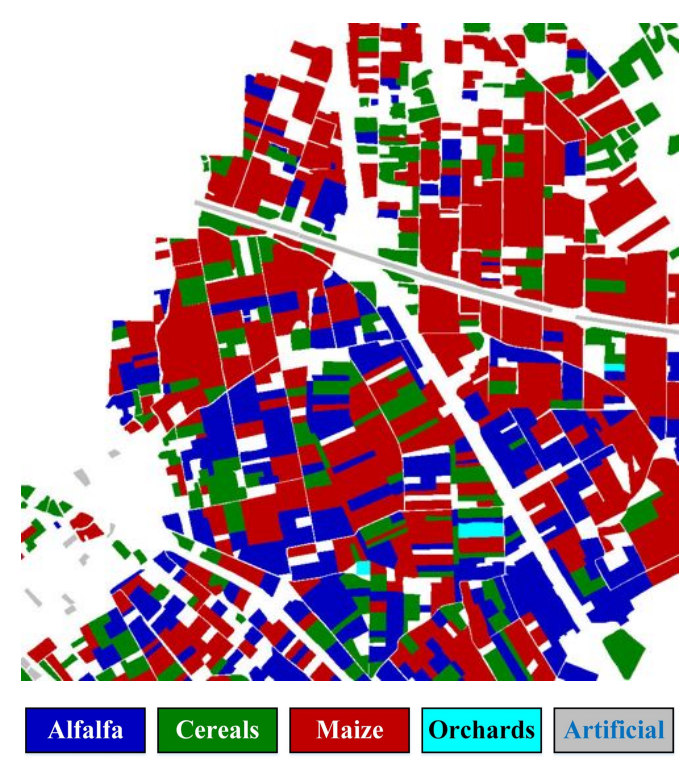

(b) Testing polygons

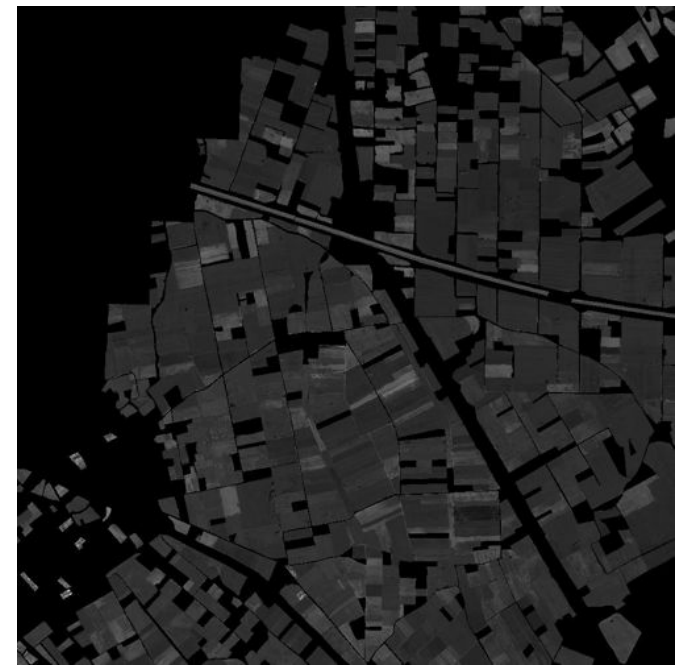

(d) (Brightness+Wetness) feature

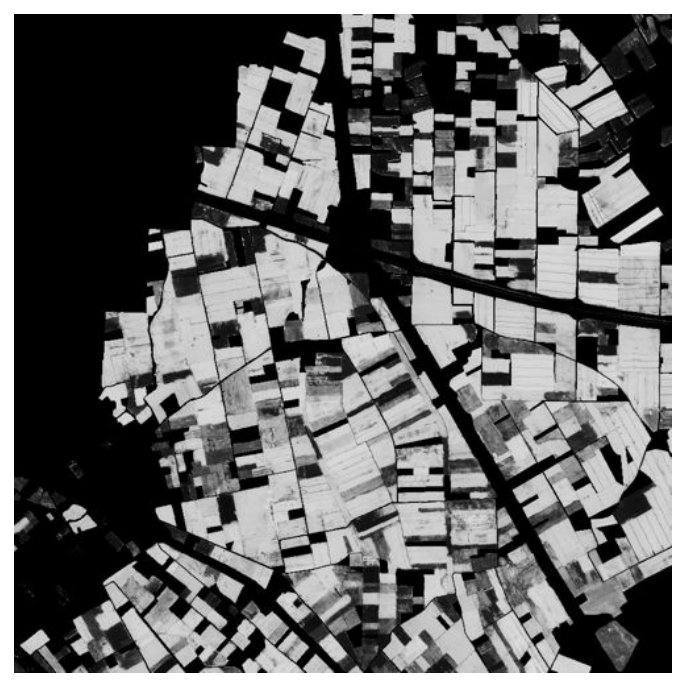

(f) NDVI of the original image

Figure 4: The extracted features used by the most significant rule produced for the maize class and the rule's activation degrees for all pixels. 


\section{Acknowledgment}

This work has been partially funded by the Andalusian Regional Government project P09-TIC-04813 and the Spanish MEC project TIN2012-38969.

This work is the result of the academic stay of the student David García in the Aristotle University of Thessaloniki. This stay was funded by the International Mobility Grant for PhD students of the University of Granada.

\section{References}

[1] H. Ishibuchi and T. Nakashima, Effect of rule weights in fuzzy rule-based classification systems, IEEE Transactions on Fuzzy Systems, 9(4):506-515, Aug 2001

[2] E. Bloedorn and R. S. Michalski, Data Driven constructive induction: A methodology and applications, Feature extraction. Constructive and Selection: a data mining perspective, 51-68, Kluwer, 1998.

[3] P. Sondhi, Feature Construction Methods: A Survey, Non-refereed survey. [Available online: http://sifaka.cs.uiuc.edu/ sondhi1/survey3. pdf]

[4] T. C. Vogelmann, Plant Tissue Optics, Annual Review of Plant Physiology and Plant Molecular Biology, 44:231-251, 1993.

[5] J. Qi, F. Cabot, M. S. Moran and G. Dedieu, Biophysical parameter estimations using multidirectional spectral measurements, Remote Sensing of Environment, 54:71-83, 1995.

[6] D. Haboudane, J. R. Miller, N. Tremblay and P. Vigneault, Indices-based approach for crop chlorophyll content retrieval from hyperspectral data, in Proceedings of the 2007 International Geoscience and Remote Sensing Symposium (IGARSS 2007), pages 3297-3300, July 23-27, Barcelona (Spain), 2007.

[7] D. García, A. González and R. Pérez, A feature construction approach for genetic iterative rule learning algorithm, Journal of Computer and System Sciences, 80:101-117, 2014.

[8] A. González and R. Pérez, Improving the genetic algorithm of SLAVE, Mathware \& Soft Computing, 16(1):59-70, 2009.

[9] D. García, A. González and R. Pérez, Overview of the SLAVE learning algorithm: A review of its evolution and prospects, International Journal of Computational Intelligence Systems, 7(6):1194-1221, Taylor \& Francis, 2014.

[10] T. Mitchell, Machine Learning, MacGraw-Hill, 1997.

[11] A. González and R. Pérez , Completeness and consistency conditions for learning fuzzy rules, Fuzzy Sets and Systems, 96:37-51, 1998.

[12] A. González and R. Pérez, An analysis of the scalability of an embedded feature selection model for classification problems, in Proceed- ings of the $11^{\text {th }}$ International Conference on Information Processing and Management of Uncertainty on Knowledge-Based Systems (IPMU 2006), pages 1949-1956, Paris (France), 2006.

[13] J. H. Horne, A tasseled cap transformation for IKONOS images, in Proceedings of the 2003 ASPRS Annual Conference (ASPRS 2003), Anchorage (Alaska), 2003.

[14] Y. Zhang and G. Hong, An IHS and wavelet integrated approach to improve pan-sharpening visual quality of natural colour IKONOS and QuickBird images, Information Fusion, 6:225234, 2005.

[15] R. M. Haralick, K. Shanmugam and I. Dinstein, Textural Features for Image Classification, IEEE Transactions on Systems, Man and Cybernetics, SMC-3:610-621, 1973.

[16] D. G. Stavrakoudis, J. B. Theocharis and G. C. Zalidis, A multistage genetic fuzzy classifier for land cover classification from satellite imagery, Soft Computing, 15:2355-2374, 2011.

[17] C. Cortes and V. Vapnik, Support-vector networks, Machine Learning, 20:273-297, 1995.

[18] L. Breiman, Random Forests, Machine Learning, 45:5-32, 2001. 